\title{
Scattering Amplitudes from Superconformal Ward Identities
}

\author{
D. Chicherin, ${ }^{1}$ J. M. Henn, ${ }^{1,2}$ and E. Sokatchev ${ }^{1,3,4}$ \\ ${ }^{1}$ PRISMA Cluster of Excellence, Johannes Gutenberg University, 55099 Mainz, Germany \\ ${ }^{2}$ Max-Planck-Institut für Physik, Werner-Heisenberg-Institut, 80805 München, Germany \\ ${ }^{3}$ LAPTh, Université Savoie Mont Blanc, CNRS, B.P. 110, F-74941 Annecy-le-Vieux, France \\ ${ }^{4}$ Theoretical Physics Department, CERN, 1211 Geneva 23, Switzerland
}

(Received 19 April 2018; revised manuscript received 18 May 2018; published 10 July 2018)

\begin{abstract}
We consider finite superamplitudes of $\mathcal{N}=1$ matter, and use superconformal symmetry to derive powerful first-order differential equations for them. Because of on-shell collinear singularities, the Ward identities have an anomaly, which is obtained from lower-loop information. We show that in the fiveparticle case, the solution to the equations is uniquely fixed by the expected analytic behavior. We apply the method to a nonplanar two-loop five-particle integral.
\end{abstract}

DOI: 10.1103/PhysRevLett.121.021602

Conformal symmetry has played a central role in quantum field theory for many decades. In recent years, its consequences are being actively explored within the AdS/CFT correspondence, the bootstrap program, and high-energy QCD.

In particle physics, scattering amplitudes are fundamental objects, relevant for collider physics. In the high-energy regime the masses can often be neglected, and the Lagrangian becomes conformal. Implications of conformal symmetry were successfully investigated in maximally supersymmetric Yang-Mills theory, based on the remarkable duality between scattering amplitudes and Wilson loops. The conformal symmetry used there is that of the dual Wilson loop. However, the native (super)conformal symmetry of the amplitudes is largely unexplored. The purpose of this Letter is to study its consequences in a broader framework.

One reason why this is a difficult problem is that putting the external particles on shell can render the symmetry anomalous. Tree-level amplitudes have a holomorphic anomaly [1-4] that arises when external particles become collinear. This mechanism is responsible for the breakdown of (super)conformal symmetry of tree-level amplitudes and of discontinuities of loop amplitudes [5-7].

The conformal symmetry of finite loop integrals and the associated anomalous Ward identities were studied in Ref. [8]. The anomaly occurs when an external lightlike momentum becomes collinear with a loop momentum. The equations provide nontrivial constraints; however, due to

Published by the American Physical Society under the terms of the Creative Commons Attribution 4.0 International license. Further distribution of this work must maintain attribution to the author(s) and the published article's title, journal citation, and DOI. Funded by SCOAP ${ }^{3}$. their second-order nature, it is in general not straightforward to solve them.

In the present Letter, we show how to obtain powerful first-order differential equations in a model of $\mathcal{N}=1$ massless supersymmetric matter. The amplitudes are infrared finite. A certain sector of the amplitudes consists of ultraviolet finite Feynman diagrams, so within it we are not affected by the running coupling. Naively, the Feynman integrals in this sector should enjoy all the symmetries of the model, in particular (super)conformal symmetry. We show that special conformal supersymmetry is broken by collinear contact terms inside the finite Feynman integrals, and we derive Ward identities that quantify this effect. We remark that a similar phenomenon was observed in the context of the supersymmetric Wilson loop in Refs. [9-11].

As a first application, we focus on the five-particle case, which is interesting for several reasons. First, it turns out that for four particles (super)conformal symmetry does not give any restrictions. So, from this point of view, five particles is the first nontrivial case. On the other hand, fiveparticle scattering at higher loops involves intricate transcendental functions [12-14] and is of considerable current interest $[15,16]$.

We show how the differential equations can be used to fully determine the answer. The fermionic generator is reduced to the so-called twistor collinearity operator of Ref. [1]. Its kernel consists of a function of holomorphic cross-ratios only [6]. We propose a way to fix this boundary freedom by imposing expected analytic properties of the function. We illustrate these ideas by computing a nontrivial nonplanar two-loop five-particle integral.

$\mathcal{N}=1$ matter superamplitudes and their superconformal anomalies.-We consider scattering amplitudes in the WessZumino model of $\mathcal{N}=1$ massless supersymmetric matter. The multiplet is described by a chiral superfield $\Phi(p, \theta)$ and 


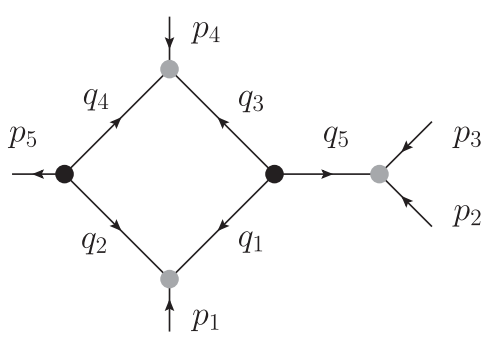

FIG. 1. One-loop $\mathcal{N}=1$ matter supergraph. Black and grey blobs denote antichiral and chiral vertices, respectively.

its antichiral conjugate $\bar{\Phi}(p, \bar{\theta})$. The Lagrangian contains cubic interactions $g \int d^{2} \theta \Phi^{3}$ and $g \int d^{2} \bar{\theta} \bar{\Phi}^{3}$.

This model is superconformal at the classical level. At the quantum level, the symmetry is broken, but only by propagator corrections, and the beta function is proportional to the anomalous dimension of the superfield [17]. This property allows us to study individual finite supergraphs, with the only requirement that they do not contain propagator correction subgraphs. See Figs. 1 and 2 for sample graphs.

Such graphs are naively superconformal. We will see that the symmetry is in fact broken by on-shell collinear effects. The latter can be controlled and give rise to powerful anomalous Ward identities.

In order to discuss superamplitudes, we introduce the onshell superstates

$$
\begin{aligned}
& \bar{\Phi}(p, \eta)=\bar{\phi}(p)+\eta \psi_{-}(p), \\
& \Psi(p, \eta)=\psi_{+}(p)+\eta \phi(p) .
\end{aligned}
$$

They depend on the lightlike momentum $p_{\alpha \dot{\alpha}}=\lambda_{\alpha} \tilde{\lambda}_{\dot{\alpha}}$, and on the Grassmann variable $\eta$. For the antichiral on-shell state $\eta=[\tilde{\lambda} \bar{\theta}] \equiv \tilde{\lambda}_{\dot{\alpha}} \bar{\theta}^{\dot{\alpha}}$; for the chiral state it is defined as the Fourier transform of $\lambda^{\alpha} \theta_{\alpha}$. The Feynman rules of the quantum theory are well known. Here we only need the mixed propagator (wave function) $\langle\bar{\Phi}(-p, \bar{\theta}) \Psi(p, \eta)\rangle=$ $\eta+[\tilde{\lambda} \bar{\theta}]$, and the antichiral cubic vertex involving a Grassmann integral, $g \int d^{2} \bar{\theta} \bar{\Phi}^{3}$.

In our $\mathcal{N}=1$ model, the breaking of conformal supersymmetry can already be seen for the three-point vertex function of two off-shell superfields and one on-shell state. Apart from illustrating the mechanism, this object will also constitute the main building block for our practical calculations. At leading order, it is given by

$$
\begin{aligned}
\mathcal{F} & \equiv\left\langle\bar{\Phi}\left(q_{1}, \bar{\theta}_{1}\right) \bar{\Phi}\left(q_{2}, \bar{\theta}_{2}\right) \mid \bar{\Phi}(p, \eta)\right\rangle_{g} \\
& =\delta^{4}(P) \delta^{2}(Q) \frac{g}{q_{1}^{2} q_{2}^{2}} .
\end{aligned}
$$

The delta functions account for momentum $P=q_{1}+q_{2}+$ $p$ and supercharge $Q=\bar{\theta}_{1} q_{1}+\bar{\theta}_{2} q_{2}+\eta \lambda$ conservation. Invariance under $\bar{Q}=\sum_{i}\left(\partial / \partial \bar{\theta}_{i}\right)+\tilde{\lambda}(\partial / \partial \eta)$ is also readily verified. We focus on the chiral superconformal

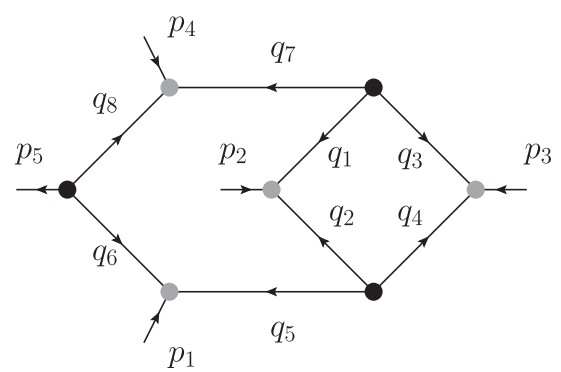

FIG. 2. Nonplanar two-loop $\mathcal{N}=1$ matter supergraph.

generator $S_{\alpha}=\frac{1}{2} \sum_{i}\left(\partial^{2} / \partial q_{i}^{\alpha \dot{\alpha}} \partial \bar{\theta}_{i \dot{\alpha}}\right)+\left(\partial^{2} / \partial \eta \partial \lambda^{\alpha}\right)$. It annihilates $\mathcal{F}$ for generic momentum configurations. However, this is not true in the collinear regime $q_{1} \sim q_{2} \sim p$. When the bosonic derivatives in $S_{\alpha}$ act on the product of propagators in Eq. (2), they generate contact terms. We postpone the details to a future publication and give the result for the collinear superconformal anomaly

$$
\begin{aligned}
S^{\alpha} \mathcal{F}= & 2 i \pi^{2} \lambda^{\alpha} \int_{0}^{1} d \xi\left(\eta+\left[\tilde{\lambda} \bar{\theta}_{1}\right] \xi+\left[\tilde{\lambda} \bar{\theta}_{2}\right] \bar{\xi}\right) \\
& \times \delta^{4}\left(q_{1}+\xi p\right) \delta^{4}\left(q_{2}+\bar{\xi} p\right),
\end{aligned}
$$

where $\bar{\xi}=1-\xi$. The antichiral generator is not anomalous, $\bar{S}_{\dot{\alpha}} \mathcal{F}=0$. The superconformal algebra $\left\{S_{\alpha}, \bar{S}_{\dot{\alpha}}\right\}=$ $K_{\alpha \dot{\alpha}}$ then yields an anomaly of the conformal generator $K$ similar to that of Ref. [8].

The anomalous Ward identity Eq. (3) tells us that when acting on an on-shell supergraph, such as the one shown in Fig. 1, we pick up an anomaly contribution from each chiral three-point vertex (grey blob) connected to an external antichiral on-shell state. Thanks to the extra delta function in Eq. (3), a loop integration is localized, so that the anomaly term for an $L$-loop graph is expressed in terms of a onefold integral of an $(L-1)$-loop graph.

Let us now apply this anomaly equation to computing $\mathcal{N}=1$ matter superamplitudes. They can be classified according to their Grassmann degree. Because of $Q$ supersymmetry, the general form of an amplitude of $m$ $\bar{\Phi}$ states and $n \Psi$ states is

$$
\mathcal{A}_{n, m}=\delta^{4}(P) \delta^{2}(Q) \mathcal{P}_{n, m} .
$$

The Grassmann degree of $\mathcal{P}_{n, m}$ is related to the $U(1) R$ symmetry of the theory. We have $R_{\eta}=1, R_{\bar{\Phi}}=2 / 3$, and $R_{\Psi}=1 / 3$. Therefore, $\mathcal{P}_{n, m}$ has $R$-charge $(n+2 m) /$ $3-2 \geq 0$. In this Letter, we consider the five-particle case $n=1$ and $m=4$, i.e., $R_{\mathcal{P}}=1$, as a first application.

The five-leg amplitudes we consider have the structure

$$
\begin{aligned}
\mathcal{A}_{1,4} & =\left\langle\bar{\Phi}\left(p_{1}, \eta_{1}\right) \bar{\Phi}\left(p_{2}, \eta_{2}\right) \bar{\Phi}\left(p_{3}, \eta_{3}\right) \bar{\Phi}\left(p_{4}, \eta_{4}\right) \Psi\left(p_{5}, \eta_{5}\right)\right\rangle \\
& =\delta^{4}(P) \delta^{2}(Q) \Xi_{123} C(p) .
\end{aligned}
$$

Here we used the $\bar{Q}$ invariant 


$$
\Xi_{123}=\eta_{1}[23]+\eta_{2}[31]+\eta_{3}[12] .
$$

Any three $\eta$ 's define such an invariant, but all the choices are equivalent due to supercharge conservation, e.g., $\Xi_{123}=(\langle 45\rangle /\langle 12\rangle) \Xi_{345}$, etc. Thus, the entire superamplitude Eq. (5) is determined by a single bosonic function $C(p)$. The latter can be found by extracting a component amplitude, for example, $[23]\langle 45\rangle C=\left\langle\psi_{-}(1) \bar{\phi}(2) \bar{\phi}(3) \psi_{-}(4) \phi(5)\right\rangle$, which corresponds to the $\eta_{1} \eta_{4} \eta_{5}$ term in Eq. (5).

Let us now turn to the superconformal Ward identities. When $S^{\alpha}$ acts on the rhs of Eq. (5), it commutes with $\delta^{4}(P) \delta^{2}(Q)$, so that we only need the relation

$$
S^{\alpha}\left[\Xi_{123} C\right]=F_{123}^{\alpha} C,
$$

where

$$
F_{123}^{\alpha}=[23] \frac{\partial}{\partial \lambda_{1 \alpha}}+[31] \frac{\partial}{\partial \lambda_{2 \alpha}}+[12] \frac{\partial}{\partial \lambda_{3 \alpha}}
$$

is the so-called twistor collinearity operator, see Ref. [1]. We remark that we could have equally well acted with $\bar{S}^{\dot{\alpha}}$ on the conjugated amplitude $\mathcal{A}_{4,1}$, which has $R_{\mathcal{P}}=0$.

In order to determine the right-hand side of the Ward identity we need to evaluate explicitly the $S$ variation of the supergraphs contributing to $\mathcal{A}_{1,4}$ (recall that we neglect propagator corrections). The chiral vertex functions are exactly $S$ invariant. The antichiral ones are invariant up to a contact term, see Eq. (3), which becomes relevant due to the loop integrations. So for $\mathcal{A}_{1,4}$ we have contributions from the antichiral legs $1,2,3,4$. The generator $S^{\alpha}$ lowers the Grassmann degree by one, so we have

$$
S^{\alpha} \mathcal{A}_{1,4}=\delta^{4}(P) \delta^{2}(Q) \sum_{i=1,2,3,4} \lambda_{i}^{\alpha} A_{i}(p)
$$

with some bosonic anomaly functions $A_{i}(p)$. Comparing with Eqs. (5) and (7), we derive the anomalous superconformal Ward identity

$$
F_{123}^{\alpha} C(p)=\sum_{i=1,2,3,4} \lambda_{i}^{\alpha} A_{i}(p)
$$

We note that the freedom in choosing the superinvariant $\Xi_{i j k}$ also affects the collinearity operator $F_{i j k}$. Thus we have $\langle 45\rangle F_{345} C=\langle 12\rangle F_{123} C$, etc., where momentum conservation is assumed. However, the additional equations do not provide new information.

It is convenient to define the dimensionless, helicityneutral function

$$
f=\langle 45\rangle[14][23] C .
$$

For general five-particle kinematics, $f$ depends on four dimensionless variables. They can be chosen as

$$
x_{1}=-1-\frac{s_{14}}{s_{15}}, \quad x_{2}=-1-\frac{s_{14}}{s_{45}}
$$

where $s_{i j}=2 p_{i} \cdot p_{j}$, and

$$
x_{3}=\frac{[12][34]}{[23][41]}, \quad x_{4}=\frac{[23][45]}{[34][52]} .
$$

The real variables $x_{1}, x_{2}$ are parity even, while the complex variables $x_{3}, x_{4}$ undergo conjugation under parity.

Let us comment on the solutions of the homogeneous equation $F_{123}^{\alpha} \tilde{f}=0$. The two components of this equation fix the dependence on $x_{1}, x_{2}$, while $F_{i j k}^{\alpha} x_{3}=F_{i j k}^{\alpha} x_{4}=0$, so that any function of the holomorphic variables $x_{3}, x_{4}$ solves the homogeneous equation, see also Ref. [6]. However, transcendental functions of these variables have unphysical branch cut properties; consequently, we expect the freedom of the homogeneous solution to reduce to just one integration constant.

Box with off-shell leg.-Let us illustrate the method with a one-loop example. We consider the box with one off-shell leg, see Fig. 1. Extracting the component $\eta_{1} \eta_{4} \eta_{5}$ we express the bosonic function $R$ as the following Feynman integral

$$
f=-[14] \int \frac{d^{4} \ell}{i \pi^{2}} \frac{\left\langle 1\left|q_{2} \tilde{q}_{4}\right| 4\right\rangle}{q_{1}^{2} q_{2}^{2} q_{3}^{2} q_{4}^{2}} .
$$

As is well known [18], this coincides with the "magic" pentagon integral of Ref. [19], as well as with the sixdimensional one-mass scalar box. It is given by

$$
f=\mathrm{Li}_{2}\left(x_{1} x_{2}\right)-\mathrm{Li}_{2}\left(-x_{1}\right)-\mathrm{Li}_{2}\left(-x_{2}\right)+\mathrm{Li}_{2}(1) .
$$

Let us now derive the result for $f$ from the superconformal Ward identity [Eq. (10)]. Anomalies originate from the antichiral legs 1 and 4, see Eq. (3), while $A_{2}=A_{3}=0$, up to contact terms. A short supergraph calculation gives

$A_{1}=\frac{\log \left(s_{23} / s_{45}\right)}{\langle 15\rangle\left(s_{23}-s_{45}\right)}, \quad A_{4}=\frac{\log \left(s_{23} / s_{15}\right)}{\langle 45\rangle\left(s_{23}-s_{15}\right)}$.

Projecting the two components of Eq. (10) with independent spinors, we find

$$
\begin{aligned}
& x_{1} \partial_{x_{1}} f\left(x_{1}, x_{2}\right)=\log \frac{1+x_{1}}{1-x_{1} x_{2}} \equiv a_{1}\left(x_{1}, x_{2}\right), \\
& x_{2} \partial_{x_{2}} f\left(x_{1}, x_{2}\right)=\log \frac{1+x_{2}}{1-x_{1} x_{2}} \equiv a_{4}\left(x_{1}, x_{2}\right) .
\end{aligned}
$$

It is clear from Fig. 1 that the functions $f, a_{1}$, and $a_{4}$ are independent of $x_{3}, x_{4}$.

The system of differential equations, Eq. (17), determines $f$ up to an arbitrary constant. The latter can be fixed by demanding finiteness of $C$ in Eq. (11) as $s_{14} \rightarrow 0$. The final solution takes the form

$$
f=\int_{-1}^{x_{1}} \frac{d t}{t} a_{1}\left(t, x_{2}\right)=\int_{-1}^{x_{2}} \frac{d t}{t} a_{4}\left(x_{1}, t\right) .
$$

To see that each of these integrals solves both equations in Eq. (17), one uses the integrability condition $x_{2} \partial_{x_{2}} a_{1}-$ $x_{1} \partial_{x_{1}} a_{4}=0$ and the property $a_{1}\left(x_{1},-1\right)=a_{4}\left(-1, x_{2}\right)=0$ 
of the anomaly terms. It is easy to check that Eq. (18) agrees with Eq. (15).

We find it amusing to note that, although the superconformal Ward identities are trivial for four particles, one can nonetheless obtain the result for the four-particle amplitude by taking the (finite) collinear limit $p_{2} \sim p_{3}$ of our result, which yields $\log ^{2} s_{15} / s_{45}+\pi^{2}$.

Application to nonplanar five-particle integral.-Next, we consider the nonplanar pentabox diagram of Fig. 2. Its $\eta_{1} \eta_{4} \eta_{5}$ component is given by

$f=\frac{-[14][23]}{\left(i \pi^{2}\right)^{2}} \int \frac{d^{4} \ell_{1} d^{4} \ell_{2}}{q_{1}^{2} \ldots q_{8}^{2}}\left\langle 2\left|q_{2} \tilde{q}_{4}\right| 3\right\rangle\left\langle 1\left|q_{5} \tilde{q}_{7}\right| 4\right\rangle$.

The symbol [20] of this integral was recently obtained using the bootstrap approach [14]. Here we compute the full function, in a straightforward way, using our new method. In this way, we also prove the result of Ref. [14], without any assumptions about the symbol alphabet.

The anomalous superconformal Ward identity for this integral involves four anomaly terms $A_{i}$ corresponding to the four chiral vertices in Fig. 2. Because of the $1 \leftrightarrows 4$ and $2 \leftrightarrows 3$ symmetries, only two of them are independent. The delta functions in Eq. (3) reduce the $A_{i}$ to one-loop integrals. $A_{1}$ is determined by the one-mass box with the magic numerator computed above; $A_{2}$ involves a sum of two pentagons with one massive corner.

Using known expressions for these one-loop functions, we find

$$
\begin{aligned}
A_{1}= & \frac{1}{\langle 15\rangle} \int_{0}^{1} \frac{d \xi}{\xi_{s_{45}}+\bar{\xi}_{s_{23}}}\left[\operatorname{Li}_{2}\left(1-\frac{\xi_{s_{45}}+\bar{\xi}_{s_{23}}}{\xi s_{12}}\right)\right. \\
& \left.+\operatorname{Li}_{2}\left(1-\frac{\xi_{s_{45}}+\bar{\xi}_{s_{23}}}{\xi_{s_{13}}}\right)+\frac{1}{2} \log ^{2}\left(\frac{s_{12}}{s_{13}}\right)+\frac{\pi^{2}}{6}\right] \\
A_{2}= & -[25] \int_{0}^{1} \frac{d \xi}{\langle 51\rangle[13]\langle 32\rangle[25]+\bar{\xi}_{s_{23} s_{25}}} \\
& \times \log \frac{\xi \bar{\xi}_{s_{12} s_{24}}}{\left(\xi_{s_{15}}+\bar{\xi}_{\left.s_{34}\right)\left(\xi_{s_{13}}+\bar{\xi}_{s_{45}}\right)}\right.} \\
& \times \log \frac{\bar{\xi}_{s_{45}\left(\xi_{s_{15}}+\bar{\xi}_{s_{34}}\right)}}{\xi_{s_{15}\left(\xi_{s_{13}}+\bar{\xi}_{s_{45}}\right)}}
\end{aligned}
$$

Integrating over $\xi$, we find that the anomaly equation, Eq. (10) takes the form

$$
F_{123}^{\alpha} f=[14][23]\langle 45\rangle \sum_{i=1}^{4} \lambda_{i}^{\alpha} \frac{a_{i}}{r_{i}}
$$

where $a_{i}$ are pure functions of weight three, and where

$$
\begin{aligned}
& r_{1}=\langle 15\rangle\left(s_{23}-s_{45}\right), \quad r_{2}=\langle 25\rangle s_{23}, \\
& r_{3}=\langle 35\rangle s_{23}, \quad r_{4}=\langle 45\rangle\left(s_{23}-s_{15}\right) .
\end{aligned}
$$

Projecting Eq. (21) onto its independent components, we can write

$$
\begin{aligned}
\tilde{d} f= & a_{1} \tilde{d} \log x_{1}+a_{4} \tilde{d} \log x_{2} \\
& +a_{2} \tilde{d} \log \frac{1-x_{1} x_{2}}{\left(1+x_{2}\right)\left(x_{3}-1\right) x_{4}+\left(1+x_{1}\right)\left(x_{3} x_{4}-1\right)} \\
& +a_{3} \tilde{d} \log \frac{1-x_{1} x_{2}}{\left(1+x_{2}\right) x_{3} x_{4}+\left(1+x_{1}\right)\left(x_{3} x_{4}-1\right)},
\end{aligned}
$$

with $\tilde{d}=d x_{1} \partial_{x_{1}}+d x_{2} \partial_{x_{2}}$. We note that the integrability condition $\tilde{d}^{2} f=0$ yields a nontrivial cross-check on the calculation of the anomaly terms $a_{i}$ appearing in Eq. (23). Equation (23) determines $f$ up to an arbitrary function $g\left(x_{3}, x_{4}\right)$. We now give two ways of fixing the latter.

One method follows the one-loop example above. Using a Feynman parameter representation of the integral Eq. (19), one can show that $\lim _{s_{14} \rightarrow 0} f=f\left(x_{1}=x_{2}=-1\right)=0$, while keeping $x_{3}, x_{4}$ constant, i.e., $g\left(x_{3}, x_{4}\right)=0$.

This remarkable fact is not a coincidence and leads us to a second method of fixing the boundary data. Imagine that we have already found the correct solution for $f$. We argue that any additional, nontrivial function $g\left(x_{3}, x_{4}\right)$ would introduce unphysical analytic behavior, such as branch cuts depending on the holomorphic variables $x_{3}, x_{4}$ only. This argument also explains the existence of some values of $x_{1}$, $x_{2}$ [in our case $(-1,-1)$ ], for which $f$ and hence $g$ are independent of $x_{3}, x_{4}$.

We can write the solution for $f$ by integrating, e.g., $\partial_{x_{1}} f$ from the boundary point $x_{1}=-1$, in close analogy with Eq. (18), but this time with contributions from $a_{1}, a_{2}, a_{3}$. To see this, one notices that the parameter representations [Eq. (20)] satisfy $a_{1}\left(x_{1},-1, x_{3}, x_{4}\right)=a_{4}\left(-1, x_{2}, x_{3}, x_{4}\right)=0$. For convenience, we focus on the kinematic region $s_{i, i+1}<0, s_{13}<0, s_{24}<0$, for which $f$ is real-valued. Some care is required, as the boundary point $x_{1}=-1$ lies outside the above region. The integration is performed analytically using Ref. [21] and evaluates to weight four multiple polylogarithms [22]. The final expression is given in Supplemental Material [23].

In principle, one could rewrite the answer in terms of a minimal function basis, see Ref. [20]. We have checked that it involves nonclassical polylogarithms, e.g., $\mathrm{Li}_{2,2}$, in addition to classical polylogarithms. We leave this rewriting and the study of suitable function arguments [24] for future work.

Let us discuss checks of our result. The symbol of the result agrees with Ref. [14]. The function has discontinuities [22] only at expected values of the $s_{i j}$, and not at values depending on the holomorphic variables $x_{3}, x_{4}$. Finally, we compared the numeric evaluation [25] to that of an integral representation.

Let us study the symbol of our answer in light of the structure suggested by the differential equation, Eq. (23), which constrains the possible last entries. Four of the last 
entries simply correspond to the arguments of the logarithms of Eq. (23). In terms of the $\left\{W_{i}\right\}$ alphabet, $i=$ $1, \ldots, 31$ of Ref. [14], they are given by $W_{14} / W_{5}, W_{2} / W_{20}$, $W_{2} / W_{18}$ and $W_{12} / W_{4}$. Other last entries must be functions of $x_{3}, x_{4}$ only. There are only 5 such letters in the alphabet of Ref. [14], namely, $W_{5} W_{17} W_{26} /\left(W_{1} W_{4}\right)$, and cyclic. We find that the symbol of our answer can indeed be written in terms of eight of those nine last entries.

Discussion.-In this Letter we took first steps towards finding implications of superconformal symmetry for looplevel amplitudes. We derived powerful superconformal Ward identities for finite scattering amplitudes in a model of $\mathcal{N}=$ 1 supersymmetric matter. The essential reason for the presence of the anomaly lies in the on-shell external legs, which makes singular collinear configurations possible.

We obtained a first-order differential equation for a given $L$-loop integral, with the right-hand side expressed as a single parameter integral over certain $(L-1)$-loop integrals. This is to be contrasted with the traditional differential equations approach (see, e.g., Ref. [26]), which involves a large system of equations, and whose generation typically requires considerable computer algebra. Being first order, our equations are very powerful. Their kernel is easily seen to contain only holomorphic functions. We argued that the absence of unphysical analytic behavior is enough to fix this freedom.

It is important to note that our method applies equally to planar and nonplanar amplitudes. We illustrated this by evaluating a nonplanar two-loop five-particle Feynman integral.

The differential equations shed light on the class of special functions needed. This information can be valuable input for "bootstrap" approaches that have been successfully used in several cases, such as amplitudes in $\mathcal{N}=4$ super Yang-Mills theory [27,28] and rapidity anomalous dimensions [29]. Additionally, we expect that the classes of special functions defined by our differential operators will be of interest to mathematicians.

The class of integrals considered here is generated by $\mathcal{N}=$ 1 matter supergraphs without propagator corrections. An avenue for future research is to extend the present method to include $\mathcal{N}=1$ gauge superfields to cover the full spectrum of massless particles. We expect that this will allow the application of the method to the "local" integrals for scattering amplitudes in maximally supersymmetric Yang-Mills theory [19]. This will also enable us to elucidate the relationship with the powerful Wilson loop approach of Refs. [9-11] and seek applications to more general amplitudes.

We are grateful to Simon Caron-Huot for stimulating discussions. The authors were supported in part by the PRISMA Cluster of Excellence at Mainz university. This project has received funding from the European Research Council (ERC) under the European Union's Horizon 2020 research and innovation programme (Grant Agreement No. 725110), "Novel structures in scattering amplitudes."

[1] E. Witten, Commun. Math. Phys. 252, 189 (2004).

[2] F. Cachazo, P. Svrcek, and E. Witten, J. High Energy Phys. 10 (2004) 077.

[3] F. Cachazo, arXiv:hep-th/0410077.

[4] S. J. Bidder, N. E. J. Bjerrum-Bohr, L. J. Dixon, and D. C. Dunbar, Phys. Lett. B 606, 189 (2005).

[5] T. Bargheer, N. Beisert, W. Galleas, F. Loebbert, and T. McLoughlin, J. High Energy Phys. 11 (2009) 056.

[6] G. P. Korchemsky and E. Sokatchev, Nucl. Phys. B832, 1 (2010).

[7] N. Beisert, J. Henn, T. McLoughlin, and J. Plefka, J. High Energy Phys. 04 (2010) 085.

[8] D. Chicherin and E. Sokatchev, J. High Energy Phys. 04 (2018) 082.

[9] S. Caron-Huot, J. High Energy Phys. 12 (2011) 066.

[10] M. Bullimore and D. Skinner, arXiv:1112.1056.

[11] S. Caron-Huot and S. He, J. High Energy Phys. 07 (2012) 174.

[12] T. Gehrmann, J. M. Henn, and N. A. Lo Presti, Phys. Rev. Lett. 116, 062001 (2016); [Phys. Rev. Lett. 116, 189903(E) (2016)].

[13] C. G. Papadopoulos, D. Tommasini, and C. Wever, J. High Energy Phys. 04 (2016) 078.

[14] D. Chicherin, J. Henn, and V. Mitev, J. High Energy Phys. 05 (2018) 164.

[15] S. Badger, C. Brønnum-Hansen, H. B. Hartanto, and T. Peraro, Phys. Rev. Lett. 120, 092001 (2018).

[16] S. Abreu, F. F. Cordero, H. Ita, B. Page, and M. Zeng, arXiv: 1712.03946.

[17] S. Ferrara, J. Iliopoulos, and B. Zumino, Nucl. Phys. B77, 413 (1974).

[18] L. J. Dixon, J. M. Drummond, and J. M. Henn, J. High Energy Phys. 06 (2011) 100.

[19] N. Arkani-Hamed, J. L. Bourjaily, F. Cachazo, and J. Trnka, J. High Energy Phys. 06 (2012) 125.

[20] A. B. Goncharov, M. Spradlin, C. Vergu, and A. Volovich, Phys. Rev. Lett. 105, 151605 (2010).

[21] E. Panzer, Comput. Phys. Commun. 188, 148 (2015).

[22] A. B. Goncharov, arXiv:math/0103059.

[23] See Supplemental Material at http://link.aps.org/ supplemental/10.1103/PhysRevLett.121.021602 for the analytic expression of the integral Eq. (19).

[24] C. Duhr, H. Gangl, and J. R. Rhodes, J. High Energy Phys. 10 (2012) 075.

[25] J. Vollinga and S. Weinzierl, Comput. Phys. Commun. 167, 177 (2005).

[26] M. Argeri and P. Mastrolia, Int. J. Mod. Phys. A 22, 4375 (2007).

[27] L. J. Dixon, J. M. Drummond, and J. M. Henn, J. High Energy Phys. 11 (2011) 023.

[28] S. Caron-Huot, L. J. Dixon, A. McLeod, and M. von Hippel, Phys. Rev. Lett. 117, 241601 (2016).

[29] Y. Li and H. X. Zhu, Phys. Rev. Lett. 118, 022004 (2017). 